1895] TRANSITIVE SUBSTITUTION GROUPS OF DEGREE $12 . \quad 255$

The statements made are easy consequences of the decompositions

for $p=1$ :

$$
[\epsilon]=[\zeta]+[\eta] \quad[\epsilon] \neq[0]
$$

$$
\begin{aligned}
& {\left[\begin{array}{l}
0 \\
1
\end{array}\right]=\left[\begin{array}{l}
0 \\
0
\end{array}\right]+\left[\begin{array}{l}
0 \\
1
\end{array}\right]=\left[\begin{array}{l}
1 \\
1
\end{array}\right]+\left[\begin{array}{l}
1 \\
0
\end{array}\right],} \\
& {\left[\begin{array}{l}
1 \\
1
\end{array}\right]=\left[\begin{array}{l}
1 \\
0
\end{array}\right]+\left[\begin{array}{l}
0 \\
1
\end{array}\right]=\left[\begin{array}{l}
1 \\
1
\end{array}\right]+\left[\begin{array}{l}
0 \\
0
\end{array}\right],}
\end{aligned}
$$

that is, every one-rowed mark not [0] decomposes in one way into two even characteristics (in two ways, if order of components be material) and in one way into an even and an odd characteristic.

We may treat similarly the equations

$$
\left(3^{\prime \prime}\right) \quad x_{p}=g_{p-1}, \quad\left(3^{\prime \prime \prime}\right) z_{p}=g_{p-1}+u_{p-1},
$$

and thus obtain the three determinations of Theorem III independently of one another and each in this highly satisfactory way.

The University of Chicago, June 17, 1895.

\title{
NOTE ON THE TRANSITIVE SUBSTITUTION GROUPS OF DEGREE TWELVE.
}

BY G. A. MIULER, PH.D.

§1. Primitive groups.

IN the Comptes Rendus, vol. 75, page 175\%, Camille Jordan states that there are three primitive groups of degree twelve, excluding the groups which contain the alternating group. In working over this region lately we found the following four multiply transitive primitive groups of this degree, excluding the two groups containing the alternating group. Since all such groups include transitive groups of degree eleven, we may prove that the following list is exhaustive by proving that no more than four such groups could be based upon these transitive groups. This may be done as follows:

No group can be based upon

(abcdefghijk)cyc*,

* A list of the transitive groups of degree eleven is given by Professor CouE, Quarterly Journal of Mathematics, vol. 27, p. 49. 
since such a group would contain eleven substitutions of degree twelve, and, by Sylow's theorem, at least one of these would be of order two and two of order three. Since no power of a substitution of order eleven could transform these into themselves, there would be at least eleven substitutions of order two and twenty-two of order three, which is impossible.

Since

$$
(a b c d e f g h i j k) \operatorname{cyc}(b k . c j . d i . e h \cdot f g)
$$

is half negative, the proof just given applies also to this case.

All the other transitive groups of degree eleven contain substitutions of the type

$$
\text { (abcde . fghij)cyc. }
$$

In the groups of degree twelve such substitutions would be transformed into themselves by twice as many substitutions of the group as in the corresponding groups of degree eleven. Hence it is only necessary to prove that no more such transforming substitutions are otherwise allowable than those which give rise to a single group in each case. That there is only one group of order 1320 follows directly from the facts that there is only one group of order 660 and degree twelve, and that the substitutions of

$$
\text { (abcdefghijk)cyc (bceifkjhdg)cyc }
$$

are fully determined as soon as the substitution of order eleven is given.

The group of order 660 and degree 12.

The group of order 660 and degree 11 has twelve subgroups of order 11.* If we represent these twelve subgroups by twelve letters, then if we transform the group with respect to its substitutions each of them will permute these letters in a different way, or lead to a different substitution of the twelve letters; $\uparrow$ for, suppose that the transformation with respect to the substitutions $s$ and $s^{\prime}$ would lead to the same substitution of these letters; then $s s_{a}$ and $s^{\prime} s_{a}$ would also have this property, and there would be at least two substitutions which would transform the twelve subgroups into themselves, which is impossible. Hence there is a simple group of order 660 and

* Cf. Professor Cole, Quarterly Journal of Mathematics, vol. 27, p. 47 .

+ Cf. Professor Moone, Bulletin of the American Mathematical Society, 2 d series, vol. 1, p. 61 . 
1895] TRANSITIVE SUBSTITUTION GROUPS OF DEGREE $12 . \quad 25 \%$

degree 12 which is simply isomorphic to the given group of order 660 and degree 11. This group is generated by

$$
\text { (abcdefghijk)(bdjfe .cghki)(aledkijghfb). }
$$

The group of order 1320 and degree 12.

From

and

$$
\begin{aligned}
& \text { bceifkjhdg-1 abcdefghijk bceifkjhdg = acegikbdfhj } \\
& =a b c d e f g h i j k^{2}, \\
& \text { bceifkjhdg bdjfe.chhki bceifkjhdg= bdjfe.cghki, } \\
& \text { bceifkjhdg-1 aledkijghfb bceifkjhdg=aligifhbdkc } \\
& \left.=\text { aledkijghfb(bdjfe.cghki.abcdefghijk }{ }^{2}\right)^{4},
\end{aligned}
$$

bceifkjhdg $g^{8}=b d j f e . c g h k i$,

it follows directly that bceifkjhdg may be used to generate a tail of 660 substitutions to the group just found. We may therefore write the group of order 1320 and degree 12 as follows:

$$
\text { abcdefghijk . bdjfe . cghki . aledkijghfb . bceifljhdg. }
$$

The group of order 7920 and degree 12.

This group may be obtained from the group of order 7920 in eleven letters in the same manner as we obtained the 660 . It is simply isomorphic to the given group of order 7920, and its elements correspond to the subgroups of order 660 in this group. The generating substitutions are

$$
a b i e g d h f c j k \text {. aegci.bfjhd . ac.eg . bd . fh . acifgbhkljd. }
$$

The group of order 95040 and degree 12.

This is the well-known fivefold transitive group of twelve letters. It is generated by

$$
\text { abiegdhfcjk . aegci . bfjhd . abcd . efgh . abdihfjcelkl. }
$$

\section{§ 2. Simple non-primitive group.}

The substitutions of a non-primitive group which do not interchange the systems form a self-conjugate subgroup.* If this self-conjugate subgroup reduces to identity, it may happen that the non-primitive group is simple. The first instance of this kind occurs in the non-primitive groups of degree twelve.

* Jordan, Traité des Substitutions, Art. 62. 
The group given on page 106 of Netto's Theory of Substitutions (American edition) as an instance that " non-primitivity may occur in a simple group" is not simple, as it involves negative substitutions. This group might well be replaced by the group of order 60 and degree 12, which is generated by

$$
a c i \cdot b d j \cdot e g k \cdot f h l \cdot a c f j l \cdot b d e i k,
$$

since this is actually a simple non-primitive group, and is the simplest instance of this kind. It is simply isomorphic to the alternating group of degree five.*

§3. Non-primitive groups based upon the second simple isomorphism of an alternating and symmetric group to itself.

The simplest bases for non-primitive groups are obtained by making a group simply isomorphic to itself by merely writing after the separate substitutions of the group the same substitutions in a different set of letters. It may happen that a group can be made simply isomorphic to itself in a way which cannot be obtained from this group by transformation. Among the alternating and symmetric groups this is only possible if the group is of degree six. This single exception may be associated with the single exception in which the alternating group is not simple and the symmetric group has a self-conjugate subgroup besides the alternating group, viz., for degree four. The three non-primitive groups of degree twelve, which are based upon a second simple isomorphism of the alternating and symmetric groups with themselves are, therefore, the only instances of this kind that can occur. These groups may be written as follows:

$$
\begin{aligned}
& 720(a b c d e f \cdot g h i j k l) \operatorname{pos}_{2}\{a g \cdot b h \cdot d i \cdot c j \cdot e k \cdot f l, \\
& 1440(a b c d e f \cdot g h i j k l) a_{2}(a g . b h . d i \cdot c j . e k \cdot f l) .
\end{aligned}
$$

The second simple isomorphisms of these basal groups are obtained by making the symmetric and alternating groups of degree five correspond to the other primitive subgroups of orders 120 and 60 respectively.

UNIVERsity OF MichigaN, May 31, 1895.

* Cf. Drck, Mathematische Annalen, vol. 22, p. 108. 